\title{
$\sqrt{ }$ laboratory result management: end-to-end process using automation and manual call centre
}

${ }^{1}$ Department of Anaesthesia, Yong Loo Lin School of Medicine, National University Health System, Singapore, Republic of Singapore 2Department of Laboratory Medicine, Yong Loo Lin School of Medicine, National University Health System, Singapore, Republic of Singapore

${ }^{3}$ Department of Cardiology, Yong Loo Lin School of Medicine, National University Health System, Singapore, Republic of Singapore

Correspondence to Dr Lian Kah Ti, Department of Anaesthesia, Yong Loo Lin School of Medicine, National University Health System, 5 Lower Kent Ridge Road, Singapore 119074, Republic of Singapore;

lian_kah_ti@nuhs.edu.sg

Accepted 3 April 2012 Published Online First 3 May 2012

\section{ABSTRACT}

Background: Timely reporting and acknowledgement are crucial steps in critical laboratory results (CLR) management. The authors previously showed that an automated pathway incorporating short messaging system (SMS) texts, auto-escalation, and manual telephone back-up improved the rate and speed of physician acknowledgement compared with manual telephone calling alone. This study investigated if it also improved the rate and speed of physician intervention to CLR and whether utilising the manual back-up affected intervention rates.

Methods: Data from seven audits between November 2007 and January 2011 were analysed. These audits were carried out to assess the robustness of CLR reporting process in the authors'

institution. Comparisons were made in the rate and speed of acknowledgement and intervention between the audits performed before and after automation. Using the automation audits, the authors compared intervention data between communication with SMS only and when manual intervention was required.

Results: 1680 CLR were reported during the audit periods. Automation improved the rate $(100 \%$ vs $84.2 \% ; p<0.001$ ) and speed (median $12 \mathrm{~min}$ vs 23 min; $p<0.001$ ) of CLR acknowledgement. It also improved the rate $(93.7 \%$ vs $84.0 \%, p<0.001)$ and speed (median 21 min vs 109 min; $p<0.001$ ) of CLR intervention. From the automation audits, the use of SMS only did not improve physician intervention rates.

Discussion: The automated communication pathway improved physician intervention rate and time in tandem with improved acknowledgement rate and time when compared with manual telephone calling. The use of manual intervention to augment automation did not adversely affect physician intervention rate, implying that an end-to-end pathway was more important than automation alone.

\section{INTRODUCTION}

Timely and reliable communication of critical laboratory results (CLR) to the correct healthcare provider is a crucial step in reducing the potential for serious harm to the patient. ${ }^{1}$ Initiatives to improve reporting of CLR have been supported by legislative and accrediting bodies requiring hospitals to adopt workflows that ensure that CLR are communicated in a timely fashion, and that the healthcare provider acknowledges being informed. ${ }^{2-4}$ As a result, many laboratories worldwide have initiated innovative methods to improve timely CLR reporting and acknowledgement, primarily by utilising advances in medical information technology. ${ }^{5-9}$

In our institution, a dedicated call centre was set up in mid-2006 to facilitate the reporting of CLR to the responsible physician. Although this system was effective, it was workforce-intensive and prone to delays during peak periods, especially when the responsible physician was unavailable to answer the telephone. In 2008, our institution introduced automation and an end-to-end CLR management strategy to improve on the timeliness and efficiency of CLR communication. Our laboratory recently published our experience in the use of this automated communication pathway. ${ }^{10}$ With this system, we were able to achieve a $100 \%$ physician acknowledgement rate of CLR, at a median time of 16 min and a mean time of 9 min for inpatients, and a median time of $7 \mathrm{~min}$ and a mean time of 9 min for outpatients.

However, acknowledgement time and rate are only surrogate measures of the true 
desired outcome. The true desired outcome is that the physician acted appropriately upon the CLR in a timely manner. In a recent study of an automated laboratory reporting system, Singh et al found that the rates of physician follow-up of abnormal test results did not differ between those who acknowledged and those who did not acknowledge these results, concluding that acknowledgement of the receipt of test result does not automatically result in timely follow-up. ${ }^{11}$ Therefore, we decided to investigate if an automated communication pathway for CLR reporting that has been previously shown to improve timely communication of CLR to physicians when compared with a manual reporting system would also improve physician intervention rates.

In addition, we know that all automated communication pathways still require a fall-back system in instances when there is no acknowledgement from the care giver; typically, this involves manual telephone calling. We therefore also undertook to investigate if the rate and speed of physician intervention was affected by the need for manual intervention.

\section{Methodology}

The National University Hospital Singapore is a 1000bedded tertiary referral hospital offering a full range of medical and surgical specialties. Its clinical chemistry and haematology laboratories receive over 4000 samples a day. Details on the type and thresholds of laboratory results defined as CLR in our institution have been previously published. ${ }^{10}$

Manual telephone calling by a call centre to report CLR was introduced in our institution in mid-2006. The call centre was manned at all times by trained and experienced operators who were equipped with duty rosters and contact details of all doctors in our institution. Laboratory staff would alert the call centre of any CLR, and the name and department of the responsible physician. The call centre operator would then locate the responsible physician and connect that physician by telephone to the laboratory. Details of the CLR would then be conveyed to the physician by the laboratory staff. If the operator failed to contact the responsible physician, an escalation procedure was initiated. The operator identifies the physician on call using the duty roster, and attempts to contact him or her. If this fails, the process is repeated, typically by calling progressively more senior physicians (eg, house-officer to medical officer to registrar to consultant), until the CLR was acknowledged.

The automated CLR communication pathway was launched in August 2008 and was fully implemented in our institution in January 2009. Briefly, CLR from all laboratory results are filtered based on predefined thresholds and utilising a software application that harmonises existing hospital applications (hospital information system, computerised physician order entry, laboratory information system and electronic roster), short messaging system (SMS) texts are automatically sent to the responsible physicians' mobile phones. The SMS text would indicate the CLR, the normal reference range, patient's identity and patient's location. The SMS text is initially sent to the physician identified on the ordering form, and requires that the physician responds by sending back an acknowledgement SMS text of one of three predetermined choices ( $1=$ 'I will act on the result, this is my patient'; $2=$ 'I will act on the result although this is not my patient'; or $3=$ 'I will not act on the result as this is not my patient'). If the physician acknowledges either choices 1 or 2 , the process ends there. However, if the physician fails to acknowledge within $10 \mathrm{~min}$ or acknowledges choice 3 , an automated escalation procedure will be triggered. This procedure identifies the physician on call using the duty roster, and sends the SMS text message to sequential duty doctors until an acknowledgement is obtained. Failure to obtain any acknowledgement would cause the system to default to the previous manual intervention, where the call centre operators will be informed to contact the physician by telephone and connect him or her to the laboratory staff who will convey the result verbally.

A total of seven audits were conducted to assess the robustness of our institution's CLR reporting process from November 2007 till January 2011. The audits were randomly conducted at 4-6 monthly intervals in order to accurately reflect daily practice: Healthcare providers and laboratory staff were not forewarned of the audits. Each audit was held over a period of 1 week, during which the audit team examined all interventions to all consecutive CLR by reviewing the relevant medical notes, charts and electronic medication system. During the period of study, there were no changes in documentation practices, with medical notes and charts being handwritten, while medication and investigation being on an electronic platform. The audit team consisted of nurses of the hospital's quality improvement unit. These nurses have undergone formal training to review case notes, and are primarily responsible for case note reviews for various quality improvement projects and for the JCI accreditation process. The audit team was not directly involved in the care of the audited patients. The audits were conducted as part of the patient safety initiative of the institution. As such, ethical board approval was not sought at the time when the audits were conducted. However, for the purpose of this study, we applied for and received institutional review board exemption to extract and analyse the data from the audits.

The audits collected data relating to both acknowledgement and intervention rates to CLR. For the purpose of this study, we defined interventions as 
deliberate responses to the CLR, which included instituting treatment, ordering investigations and referral to other care givers. In addition, documented inaction for reasons such as redundancy of test result, prior institution of treatment or clinical decision not to treat was also considered interventions. We excluded CLR arising from inadequate, haemolysed or contaminated samples. CLR resulting from full (complete) blood counts of oncology patients were excluded as these were deemed likely to be chronically abnormal. CLR of patients whose case notes were not available for review at the time of the audits and hence had incomplete information were also excluded.

The initial two audits were performed before the automated communication pathway was introduced; one audit was done during the transition while the latter four audits were done after. To investigate the effect of the implementation of the automated communication pathway, we compared the results of the initial two audits (manual telephone calling) with that of the latter four audits (automated communication pathway). Data relating to rate and speed of acknowledgement of CLR, and rate and speed of physician intervention were extracted. We defined acknowledgement rate as the percentage of CLR alerts that were acknowledged by a responsible physician. Acknowledgement for the manual pathway was taken as the point when the laboratory staff communicated the CLR to the physician. Acknowledgement for the automated pathway was taken as the instance when either the physician sends back the appropriate SMS response or when the laboratory staff communicated the CLR (for those that required manual intervention). Acknowledgement time was defined as the time taken from when the CLR alert was sent out to when the physician acknowledged the CLR. Intervention rate was defined as the percentage of CLR alerts with documented physician intervention. Absence of documentation was taken as failure to intervene. Intervention time was defined as the time from CLR alert to documented physician intervention. When there was no date or time stamp for an intervention, intervention time was either estimated based on supporting sources (nursing notes, case note entries or electronic entries) or was treated as missing data.

In addition, using data of the last four audits, we compared physician intervention rates and times of the CLR that were relayed solely by SMS text messages (SMS only) with those in which physicians did not respond to the SMS notifications and were then contact manually by telephone calling (manual intervention).

Statistical analysis was performed using SPSS V.17.0 (SPSS Inc.). Continuous data were analysed using unpaired $t$ test for parametric data and Mann-Whitney test for non-parametric data. Discrete data were analysed using $\chi^{2}$ test. A $p$ value of $<0.05$ was regarded as significant.

\section{RESULTS}

The total number of CLR analysed during the six audit weeks was 1680 , comprising of 412 CLR during the manual reporting periods and 1268 CLR during the automated pathway periods (table 1 ). The number of CLR reported by our laboratories steadily increased from the time of our first audit in 2007 to that of the seventh audit in 2011. In 2010, there were 21399 CLR reported or an average of 58 CLR per day. This represented just over $1 \%$ of all reported results. Inpatients accounted for $75 \%$ of the CLR, while outpatients and emergency medicine department accounted for $8 \%$ and $17 \%$ of the CLR, respectively. Abnormal acute coronary screen and

Table 1 CLR during the audit periods

\begin{tabular}{|c|c|c|c|c|c|c|c|}
\hline Audit Number & 1 & 2 & 3 & 4 & 5 & 6 & 7 \\
\hline Audit period & $\begin{array}{l}19-25 \\
\text { November } \\
2007\end{array}$ & $\begin{array}{l}14-20 \\
\text { April } \\
2008\end{array}$ & $\begin{array}{l}4-10 \\
\text { August } \\
2008\end{array}$ & $\begin{array}{l}9-15 \\
\text { February } \\
2009\end{array}$ & $\begin{array}{l}3-9 \\
\text { August } \\
2009\end{array}$ & $\begin{array}{l}4-10 \\
\text { January } \\
2010\end{array}$ & $\begin{array}{l}10-16 \\
\text { January } \\
2011\end{array}$ \\
\hline $\begin{array}{l}\text { CLR communication } \\
\text { pathway }\end{array}$ & Manual & Manual & $\begin{array}{l}\text { Mixed manual } \\
\text { and automated }\end{array}$ & Automated & Automated & Automated & Automated \\
\hline Total CLR & 210 & 202 & 188 & 309 & 329 & 309 & 321 \\
\hline Manual calls (\%) & $164(100)$ & $89(100)$ & $\begin{array}{l}\text { Not } \\
\text { meaningful }\end{array}$ & $153(58)$ & $104(47)$ & $116(51)$ & $102(46)$ \\
\hline $\begin{array}{l}\text { CLR communicated } \\
\text { directly to physician (\%) }\end{array}$ & $113(69)$ & $62(70)$ & $\begin{array}{l}\text { Not } \\
\text { meaningful }\end{array}$ & 245 (93) & $175(79)$ & $224(98)$ & $217(98)$ \\
\hline Excluded CLR (\%) & $46(21)$ & $113(55)$ & $52(27)$ & $45(14)$ & $107(32)$ & $80(25)$ & $99(30)$ \\
\hline
\end{tabular}

Figures represent numbers with percentages in brackets.

Audit 3 was held during the transition period from manual to automated pathway, which meant that some CLR were reported manually while others utilised the automated pathway. As such, the percentage of manual calls and the percentage of CLR communicated directly to physician were primarily influenced by the type of CLR; hence, these data were not meaningful in the context of this paper.

CLR, critical laboratory results. 
potassium levels were the two most common types of CLR; inpatients from internal medicine wards accounted for just over half of all CLR.

The audits conducted during the automated pathway periods showed improvements in every aspect when compared with the audits during the manual reporting periods (table 2). The introduction of the automated communication pathway improved acknowledgement rates, acknowledgement times, physician intervention rates, time to intervention and increased the percentage of CLR communicated directly to the responsible physician.

In the audits performed during the automated communication pathway period (audits 4-7), manual intervention was required in 56\% of CLR. CLR that were reported solely through SMS only resulted in similar acknowledgement rates but faster acknowledgement times as compared with CLR reporting that required manual intervention. There were no differences in physician intervention rates when compared with CLR that required manual intervention (table 3). However, time to intervention was slightly faster in the SMS only group.

\section{DISCUSSION}

The primary finding of this study is that the implementation of an automated communication pathway to improve CLR reporting also resulted in better physician intervention rates and shorter time to intervention. This validates the efforts by regulatory and accreditation bodies to promote timely CLR reporting. Current recommendations do not require laboratories to ensure that the healthcare provider acts on these critical results: they only require that the healthcare provider acknowledges the CLR. This is likely to be due to the considerably greater difficulty in tracking interventions as opposed to tracking acknowledgement. The implicit assumption in these recommendations is that healthcare providers will be responsible enough to appropriately intervene once they are informed of the critical results.

However, Singh et al published data that challenged the assumption that informing the correct physician of abnormal laboratory results would automatically translate into appropriate intervention. ${ }^{11}$ In their study, they found that physicians did not follow-up on $26.4 \%$ of abnormal laboratory results. After excluding all results that were regarded as having no impact on patient outcome, the authors sought an explanation for the lack of follow-up by interviewing the responsible physicians. In $6.8 \%$ of cases, they could not find a good reason. Most interestingly, whether physicians acknowledged the abnormal result or not did not influence the lack of follow-up; they were equally liable to fail to follow-up.

The paper by Singh et al flagged the importance of looking at physician intervention as a true measure of success of a CLR reporting programme, rather than just acknowledgement rates and speed. It was therefore important that this study found that physician intervention rates in our institution improved in tandem with acknowledgement rates after the introduction of the new automated communication pathway. We believe that the difference between our findings and those of Singh et al are due to the urgency of the laboratory results involved. Singh et al studied abnormal but less CLR, while we studied CLR. Certainly, we would expect physicians to act with more urgency when faced, for example, with a high potassium level as compared with a raised thyroid-stimulating hormone.

It is possible that the improved physician response rate and times to CLR after introduction of the automated communication pathway were primarily due to the reduction in third party relay of results. It is known that third party relays delay the accuracy, timeliness and urgency of the reporting of CLR to the responsible physician. ${ }^{12}{ }^{13}$ In our audits, the old protocol of manual telephone calling resulted in a third party being informed over $30 \%$ of the time. Typically, this would be a nurse or allied health professional taking the message down for the physician. Even if the wrong physician was reached, this would have counted as a positive acknowledgement. This may result in a delay in physician intervention to the CLR or even failure of intervention. In contrast, our current automation protocol involved a third party in $<10 \%$ of the time, although part of this reduction may have been secondary to increasing

Table 2 Comparison between the manual and automated CLR communication pathways

\begin{tabular}{llll}
\hline CLR communication pathway & Manual & Automated & p Value \\
\hline Audit numbers & $1-2$ & $4-7$ & $<0.001$ \\
Acknowledgement rate (\%) & 84.2 & 100 & $<0.001$ \\
Median acknowledgement time (min) & 23 & 12 & $<0.001$ \\
Intervention rate (\%) & 84.0 & 93.7 & $<0.001$ \\
Median intervention time (min) & 109 & &
\end{tabular}


Table 3 Comparison between CLR communicated by SMS only and those that required manual intervention (telephone calls) during the automated communication pathway periods

\begin{tabular}{|c|c|c|c|}
\hline & SMS only & $\begin{array}{l}\text { Manual } \\
\text { intervention }\end{array}$ & p Value \\
\hline Total number of CLR in audited periods & 515 & 674 & NS \\
\hline Percentage of CLR communicated directly to physician (\%) & 100 & 87.7 & $<0.001$ \\
\hline Acknowledgement rate (\%) & 100 & 100 & NS \\
\hline Median acknowledgement time (min) & 3 & 20 & $<0.001$ \\
\hline Intervention rate $(\%)$ & 93.0 & 93.6 & NS \\
\hline Median intervention time (min) & 16.0 & 23.0 & 0.017 \\
\hline
\end{tabular}

physician acceptance and familiarity of the automated system.

However, when we looked specifically at the audits after the automated pathway was started, we found that automation per se did not improve physician intervention rates, and only marginally improved time to intervention. The advantages of using automated systems to report CLR are multiple, especially for laboratories which handle large volumes of samples. These advantages include increased speed of reporting through automation, direct two-way communication with physicians, electronically captured audit trail and less stress on staff manning the call centres. Many studies have reported better physician response times and rates with a variety of automation devices such as pagers and cell phones when compared with the manual telephone call method..$^{5-10}$ Since the implementation of the automation communication pathway in our institution almost 3 years ago, we have found that it difficult to achieve more than the current $50 \%$ acknowledgement rates without manual intervention. It was therefore fortuitous that physician intervention rates did not differ between the SMS only and manual intervention groups. This implies that the communication pathway as a whole, rather than the specific use of automation, was instrumental in the improvements seen after automation was introduced.

This is further supported by the findings of two recent studies from Etchells et $a l^{1415}$ They assessed the effect of real-time automated paging on physician intervention of CLR, and no improvement in clinical management or reduction in adverse events compared with a manual telephone call system. Although the automation aspect was quite similar, there were important differences in the communication pathway adopted by Etchells et al and our pathway. First, we required two-way communication in which physicians had to acknowledge receipt of the alert within $10 \mathrm{~min}$, which reduces the chance of an alert being forgotten or delayed. In contrast, Etchells et al utilised one-way communication, in which alerts were sent to pagers and smartphones without a necessity for acknowledgement. Second, our system was fully integrated into physician workflow such that the responsible physician could be contacted at all times. This is important as the responsible physician would know the contextual relevance of the CLR and will be better equipped to appropriately respond to the result in a timely manner. As noted by Etchells et al, their system could not reliably contact the responsible physician at all times, a weakness that they have addressed after completion of their studies. Third, they did not have a call centre for manual back up. From our experience, a manual backup is essential because delays or errors can occur from mistakes in the roster, changes in call duties, or when physicians are busy performing other duties such as resuscitation or surgery. Fourth, our institution supplies and/or pays for the subscription of mobile phones to every physician working in the hospital. This removes the necessity of having to carry multiple pagers and mobile phones, which may inconvenience physicians and potentially cause confusion as to which device is beeping.

Therefore, we believe that having an end-to-end communication pathway is essential for effective CLR communication and intervention. Key features of this pathway include providing a clear list of primary and alternate physicians to call, instituting a tight time-line for acknowledgement of results, auto-escalation and direct communication with physicians (calling their personal mobile phones instead of ward or clinic land lines). In the design of the pathway, we were cognisant of the influence of human factors and ensured that the list of critical laboratory investigations were limited in number in an attempt to avoid information overload. We also engaged our physicians in developing appropriate escalation algorithm for each clinical area, including providing department-specific feedback data and involving stake-holders in refining the escalation rosters for each department. We believe that automation alone, without consideration of human factors and without the fallback of escalation to manual telephone calling, would likely not have improved physician intervention rates. 
One caveat is that we cannot discount that the better physician intervention rate and speed with the introduction of the automated communication pathway was a result of greater awareness and better documentation over time. This is because our audits only captured what was documented by physicians, and failure to document was regarded as lack of action. Furthermore, proper documentation of CLR acknowledgement and its intervention were emphasised after each audit exercise to all physicians, and so some improvement in documentation would be expected over time. However, because improvement in intervention rates was marked with the introduction of the automation communication pathway and only gradual in the last four audits, we feel that this was not the overwhelming reason for the difference. Another inherent weakness in this study is the prepost design of the study. As with all quasi-experimental study designs, lack of randomisation and unmeasured confounders may influence the findings. ${ }^{16}$

To the best of our knowledge, this is the first paper to show that the use of an automation-based communication pathway that adheres to the requirements of internationally recognised accreditation bodies resulted in better physician intervention rates and speed in tandem with better acknowledgement rates and speed. This is particularly important as acknowledgement rates and speed are much easier to track than interventions. This paper also indicates that automation alone is not sufficient; it has to be incorporated into a pathway that is end-to-end in design.

Acknowledgements The authors acknowledge Dr Sandyha Mujumdar and $\mathrm{Dr}$ Loh Tze Ping for their contributions to the design and implementation of the automated communication pathway. The authors also thank Ms Elena Leong for facilitating the extraction of the audit data.

Contributors LKT analysed and interpreted the data, and drafted and revised the paper. SBLA conceived the study idea, designed data collection tools, monitored data collection, interpreted the data, and revised the draft paper. SS designed the data collection tools, monitored data collection, interpreted the data and revised the draft paper. SKS monitored data collection and revised the draft paper. JWLY conceived and designed the study, designed the data collection tools and revised the draft paper.

Competing interests None.
Ethics approval Ethics approval was provided by National Healthcare Group, Singapore.

Provenance and peer review Not commissioned; externally peer reviewed.

\section{REFERENCES}

1. Hanna D, Griswold P, Leape LL, et al. Communicating critical test results: safe practice recommendations. Jt Comm J Qual Patient Saf 2005;31:68-80.

2. Assessement of patients (AOP): Standard AOP.5.3 and AOP.5.3.1. Joint Commission International accreditation Standards for hospitals. 4th edn. Oakbrook, IL USA: Joint Commission International, 2010:89-90. Section 1.

3. College of American Pathologist. Critical Results Notification, COM.30000 and Critical Result Read-Back COM.30100. All Common Accreditation Master Checklist, Northfield, IL, USA 2012;16.

4. International Organization for Standardization. Testing Laboratory Checklist LAFM03-MED/MI. ISO 15189:2007. Medical laboratories Particular requirements for quality and competence, Geneva, Switzerland: 2010;37.

5. Parl FF, O'Leary MF, Kaiser AB, et al. Implementation of a closedloop reporting system for critical values and clinical communication in compliance with the goals of the Joint Commission on the Accreditation of Healthcare Organizations (JCAHO). Clin Chem 2010;56:417-23.

6. Park $H$, Min WK, Lee W, et al. Evaluating the short message service alerting system for critical value notification via PDA telephones. Ann Clin Lab Sci 2008;38:149-56.

7. Piva E, Sciacovelli L, Zaninotto M, et al. Evaluation of effectiveness of a computerized notification system for reporting critical values. $A m \mathrm{~J}$ Clin Pathol 2009;131:432-41.

8. Guidi GC, Poli G, Bassi A, et al. Development and implementation of an automatic system for verification, validation and delivery of laboratory test results. Clin Chem Lab Med 2009;47:1355-60.

9. Poon EG, Kuperman GJ, Fiskio J, et al. Real-time notification of laboratory data requested by users through alphanumeric pagers. $J$ Am Med Inform Assoc 2002;9:217-22.

10. Saw S, Loh TP, Ang SB, et al. Meeting regulatory requirements by the use of cell phone text message notification with autoescalation and loop closure for reporting of critical laboratory results. Am J Clin Pathol 2011;136:30-4.

11. Singh $\mathrm{H}$, Thomas EJ, Sittig DF, et al. Notification of abnormal lab test results in an electronic medical record: do any safety concerns remain? Am J Med 2010;123:238-44.

12. Kuperman GJ, Boyle D, Jha A, et al. How promptly are inpatients treated for critical laboratory results? J Am Med Inform Assoc 1998;5:112-19.

13. Piva E, Plebani M. Interpretive reports and critical values. Clin Chim Acta 2009;404:52-8.

14. Etchells E, Adhikari NK, Cheung C, et al. Real-time clinical alerting: effect of an automated paging system on response time to critical laboratory values - a randomized controlled trial. Qual Saf Health Care 2010;19:99-102.

15. Etchells E, Adhikari NK, Wu R, et al. Real-time automated paging and decision support for critical laboratory abnormalities. BMJ Qual Saf 2011;20:924-30.

16. Harris AD, McGregro JC, Perencevich EN, et al. The use and interpretation of quasi-experimental studies in medical informatics. J Am Med Inform Assoc 2006;13:16-23. 